\title{
Abnormal Primary Tissue Collagen Composition in the Skin of Recurrent Incisional Hernia Patients
}

\author{
BRENT WHITE, M.D., CHARLES OSIER, B.S., NANA GLETSU, PH.D., LOUIS JEANSONNE, M.D., \\ MERCEDEH BAGHAI, M.D., MELANIE SHERMAN, PH.D., C. DANIEL SMITH, M.D., BRUCE RAMSHAW, M.D., \\ EDWARD LIN, D.O. \\ From the Hernia Institute, Emory Endosurgery Unit, Department of Surgery, Emory University \\ School of Medicine, Atlanta, Georgia
}

\begin{abstract}
Recurrence of incisional hernia may be as high as 50 per cent. Abnormal collagen I/III ratios have been observed within scar tissue of patients with recurrent incisional hernias. We sought to determine whether collagen composition in primary, nonscarred tissue was similarly affected in these patients. In this prospective, case-control study, nonscarred, primary abdominal wall skin and fascia biopsies were obtained in 12 patients with a history of recurrent incisional hernias and 11 control subjects without any history of hernia while undergoing abdominal laparoscopic surgery. Tissue protein expression of collagen I and III was assessed by immunohistochemistry followed by densitometry analysis. The collagen I/III ratio in skin biopsies from the recurrent hernia group was significantly less compared with control subjects $(0.88 \pm 0.01$ versus $0.98 \pm 0.04$, respectively, $P<0.05$ ). Fascia biopsies from patients with recurrent hernias was not significantly decreased in collagen I/III ratio compared with control subjects $(0.90 \pm 0.04$ versus $0.94 \pm 0.03$, respectively, $P=0.17$ ). Decreased collagen $\mathrm{I} / \mathrm{III}$ ratios within the skin of patients with recurrent hernias not involved with scar or healing tissue suggest an underlying collagen composition defect. Such a primary collagen defect, in addition to abnormal scar formation, likely plays a significant role in the pathogenesis of recurrent incisional hernias.
\end{abstract}

\begin{abstract}
NCISIONAL HERNIAS CAN BE A major complication of the 9.8 million abdominal operations that are performed in the United States each year. ${ }^{1}$ Estimates of failed primary fascial closure after laparotomy have been reported to be as high as 50 per cent. ${ }^{2}$ Abdominal incisional hernias can subsequently lead to chronic pain, bowel obstruction, or incarceration. Based on research by Luijendink et al., up to 58 per cent of nonmesh incisional hernia repairs will recur. ${ }^{3}$ Such a high recurrence rate has not only emphasized the importance of mesh repairs and continued research in development of better mesh material, but also has prompted further research on primary tissue healing mechanisms and physiology of scar formation.

Collagen is the principal component of the extracellular matrix of both primary and scar tissue. ${ }^{4}$ It is
\end{abstract}

Address correspondence and reprint requests to Edward Lin, D.O., Assistant Professor of Surgery, Department of Surgery, Emory University School of Medicine, 1364 Clifton Road, H124, Atlanta, GA 30322. E-mail: elin2@emory.edu.

This research was supported in part by a research grant from W.L. Gore \& Associates. composed of three polypeptide chains that intertwine to form a triple helix structure The variation in chain combinations account for different collagen molecules (collagen types I, II, III, and so on, reviewed in Vander-Rest et al. ${ }^{5}$ ). The collagen I/III ratio of any given tissue determines its tensile strength and mechanical stability with an increase in collagen type III leading to abnormal crosslinking of fibrils. ${ }^{6-14}$ This abnormal crosslinking ultimately reduces the tensile strength of the abdominal wall, making it more susceptible to hernia formation under increased stress.

After injury, balanced collagen maturation and degradation is a requirement for normal scar formation. Prior research has focused on the pathogenesis of incisional hernia as a disorder of collagen regeneration during wound healing. Specifically, decreases in the collagen I/III ratio in scar tissue from both skin and fascial sites have been demonstrated in several studies using both protein and mRNA assays when comparing patients with hernias with normal subjects. $2,4,13,15$ To date, comparative analysis of the collagen content of primary, nonscarred connective tissue taken from patients with recurrent incisional hernias and healthy control subjects has yet to be performed. 
In this prospective, case-control study, we assessed collagen biochemistry in abdominal wall fascia and skin biopsies obtained from patients with recurrent incisional hernias and from a control group (no history of incisional hernias). Taking full advantage of laparoscopic surgical techniques, we focused on nonscarred tissue located away from prior incisional sites to determine if there were primary defects in skin and fascial collagen that were not confounded by previous healing. Our hypothesis was that patients with recurrent incisional hernias had inherent alterations in collagen I and III deposition in skin and fascia compared with a group of patients with normal wound healing.

\section{Materials and Methods}

\section{Patients and Specimens}

A total of 32 patients consented to participate in this study. Informed consent was obtained as approved by the institution's Institutional Review Board. Five patients were excluded for known connective tissue disorder, if they were undergoing initial (nonrecurrent) hernia repair, or if they were undergoing ongoing steroid therapy. Four patients cancelled their surgical procedure. All patient medical records were reviewed for demographic data collection and to obtain relevant surgical and medical history.

During each patient's operation, a small piece of elliptical nonscarred skin was sharply excised at the site of a lateral trocar incision located well away from the site of previous surgery and scar tissue. Subsequently, a small piece of transversalis fascia was sharply excised under direct laparoscopic visualization well away from any scar, hernia defects, or other pathology. Fascia and skin specimens were then dissected free from surrounding adipose tissue and immediately fixed in 10 per cent formaldehyde.

\section{Immunohistochemistry and Microscopy}

Specimens were then embedded in paraffin, cut to $5-\mu m$-thick sections, and adhered to a slide. The slides were then deparaffinized and rehydrated. Antigen retrieval was in a citrate buffer ( $\mathrm{pH} 6)$ using an electric pressure cooker for 5 minutes at $120^{\circ} \mathrm{C}$ with cooling for 10 minutes before immunostaining. All tissues were then exposed to 3 per cent hydrogen peroxide for 5 minutes, collagen antibodies for 30 minutes, labeled polymer, horseradish peroxidase for 30 minutes, diaminobenzidine as chromogen for 5 minutes, and automation hematoxylin (Dako, Carpinteria, CA) as counterstain for 15 minutes. The primary antibody used was a commercial peroxidase conjugated, goat anti-human IgG for collagen types I and III (Santa Cruz Biotechnology, Santa Cruz, CA). Incubations were performed at room temperature and sections were washed with Tris-buffered saline buffer between incubations. Coverslipping was performed using the Tissue-Tek SCA (Sakura Finetek USA, Inc., Torrance, CA) automatic coverslipper. After staining, regions of skin (subepidermal) and fascia were imaged and captured in a high-power field $(400 \times)$ by a digital camera (Nikon Coolpix 4500, Tokyo, Japan) using Slidebook software (Intelligent Imaging Innovations, Denver, CO) based on a Zeiss Axiovert 200M inverted microscope (Carl Zeiss, Thornwood, NY).

\section{Densitometry}

Labeled collagen types I and III were quantified through densitometry measurements using ImageJ software (National Institutes of Health, Bethesda, MD). ${ }^{16}$ The entire image was selected for each tissue and converted to a 32-bit gray scale image (gray = 0.299 red +0.587 green +0$).{ }^{18}$ The image was then inverted to display labeled tissue as bright (Fig. 1). The mean gray value (the sum of the gray values of all the pixels in the selection divided by the number of pixels) was reported in calibrated units of optical density.

\section{Statistical Analysis}

Data was analyzed using $\mathrm{R}$ statistical software ( $\mathrm{R}$ Foundation, Vienna, Austria). The ratio of collagen I to collagen III density was computed for each skin and fascia specimen. Mann-Whitney tests were used to compare the collagen I/collagen III ratios between patient groups for both skin and fascia. Data is expressed as mean \pm standard error of mean. The significance level was set at $P<0.05$.

Subanalysis was then performed comparing the experimental group with the control patients who had undergone prior abdominal surgery without hernia formation. Mann-Whitney tests were again used to compare the mean collagen I/III ratios between patient groups for both skin and fascia. Data is expressed as mean \pm standard error of mean. The significance level was set at $P<0.05$.

\section{Results}

Twenty-three patients were included in the final study analysis and divided into two categories. The study group consisted of patients with a history of recurrent incisional hernia undergoing surgery $(\mathrm{n}=$ 12). The control group consisted of patients who had no clinically evident or historical incisional hernia $(\mathrm{n}=11)$. Demographic data, including age, gender, body mass index, and relevant surgical and medical history, is noted in Table 1 . There were no statistically 

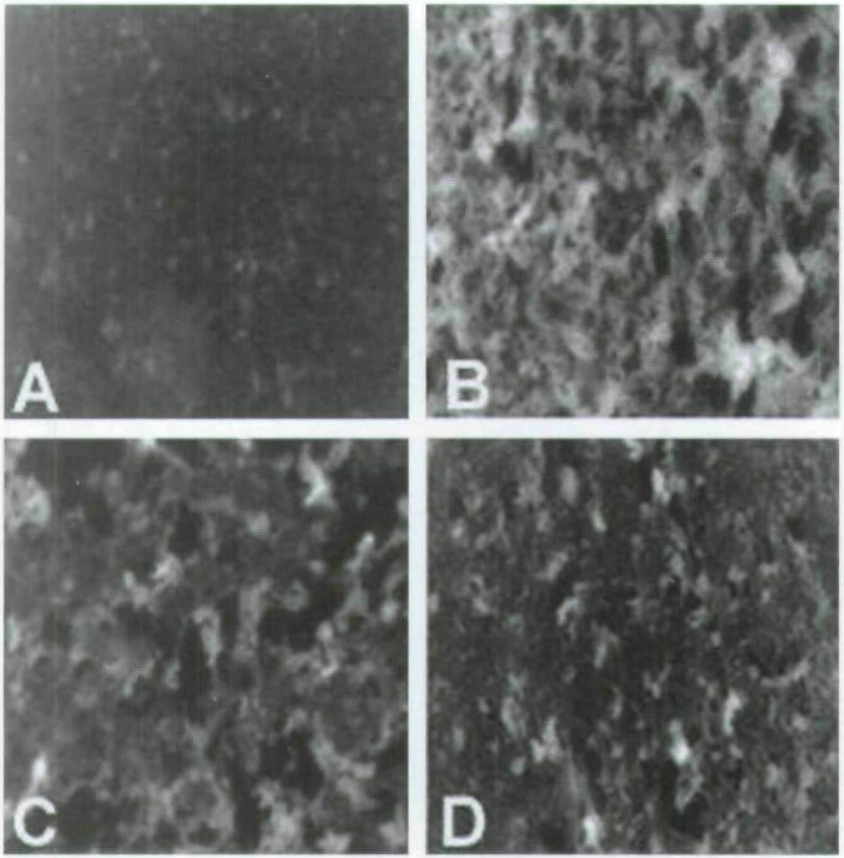

FIG. 1. IgG labeling of collagen types I and III in skin (400x, white is collagen). Note the decrease in collagen type I (A) and increase in collagen type III (B) in the tissue from a patient with a history of recurrent incisional hernia compared with control subjects (C, collagen type I, D, collagen type III).

TABle 1. Patient Demographics

\begin{tabular}{lcc}
\multicolumn{1}{c}{ Variable } & Control & Hernia \\
\hline Number & 11 & 12 \\
Men & 4 & 2 \\
Women & 7 & 10 \\
Age (years) & 51.4 & 50 \\
$\quad$ Mean & Range & $37-62$ \\
$\begin{array}{l}\text { Body mass } \\
\text { index }\left(\mathrm{kg} / \mathrm{m}^{2}\right)\end{array}$ & $30-74$ & \\
$\quad$ Mean \\
$\quad$ Range \\
$\begin{array}{l}\text { Prior abdominal } \\
\text { surgery }\end{array}$ & $22-53$ & 34.5 \\
$\quad$ Mean & & $23-47$ \\
$\quad$ Range & 1.3 & \\
$\begin{array}{l}\text { Prior } \\
\text { repair }\end{array}$ & $0-4$ & 2.6 \\
$\quad$ Mean & & $1-8$ \\
$\quad$ Range & 0 & \\
$\begin{array}{l}\text { Diabetes } \\
\text { mellitus }\end{array}$ & - & 4.7 \\
Smoke tobacco & 2 & $2-23$ \\
\hline
\end{tabular}

significant differences in age, body mass index, gender, diabetes, or tobacco use in control subjects versus patients with hernias. As expected, patients in the experimental group with recurrent incisional hernias had experienced more abdominal surgery compared with control subjects $(P<0.05)$.

Differences in collagen type staining between control subjects and patients with hernias were visually evident on a microscopic level (Fig. 1). Analysis of optical density collagen I/III ratios in skin and fascia samples found that skin biopsies obtained from patients with hernias had significantly lower collagen I/III ratios compared with skin biopsies obtained from control subjects $(0.88 \pm 0.01$ versus $0.98 \pm 0.04, P<$ 0.05 , Fig. 2). When comparing collagen $\mathrm{I} / \mathrm{III}$ ratios in fascial samples biopsied from patients with hernias versus control subjects, lower ratios were found in samples obtained from patients with hernias, although this difference was not statistically significant $(0.90 \pm$ 0.04 versus $0.94 \pm 0.03, P=0.17$, Fig. 2 ).

Subanalysis of the experimental group $(n=12)$ compared with the control subjects who had undergone prior abdominal surgery without hernia formation $(\mathrm{n}=6)$ did not alter the findings noted in our main analysis or their significance for skin $(0.88 \pm$ 0.01 versus $1.00 \pm 0.05, P=0.02)$ or fascia $(0.90 \pm$ 0.04 versus $0.94 \pm 0.03, P=0.25$ ).

\section{Discussion}

This study shows a decreased collagen I/III ratio in primary skin and fascia biopsies of patients with recurrent incisional hernias when compared with their normal counterparts. Skin samples obtained from patients with hernias had significantly lower collagen I/III ratios when compared with control subjects. Although not significant, fascia biopsies obtained from patients with hernias versus control subjects also had a lower collagen $\mathrm{I} / \mathrm{III}$ ratio.

In normal tissue, collagen I and collagen II maintain a relatively constant ratio. Previous studies have demonstrated abnormalities in the ratio of these collagen molecules in patients with a number of genetic conditions known to predispose to hernia formation such as

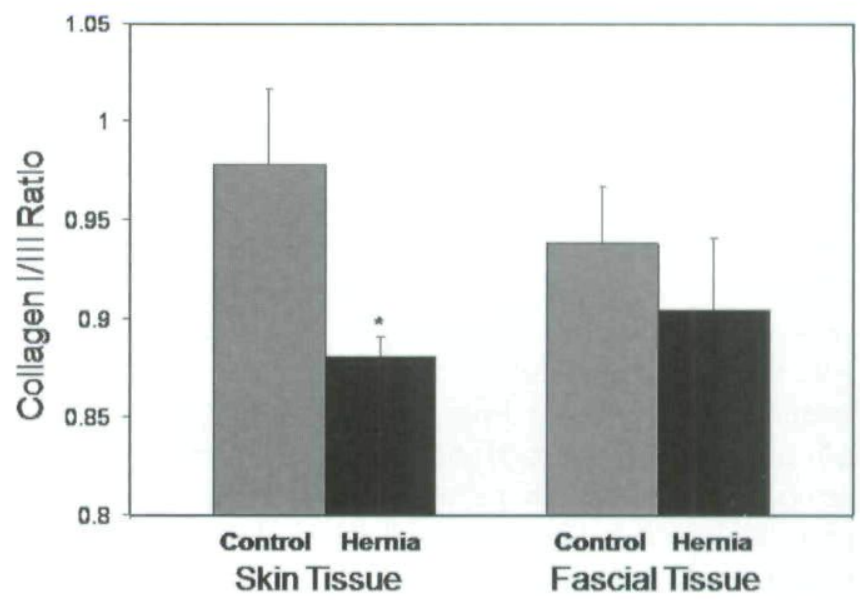

FIG. 2. Subepidermal (skin) and transversalis fascia collagen I/III ratio (ratio of optical density of collagen type I to optical density of collagen type III in a high-power field). The data are presented as mean values with standard error, $\left({ }^{*} P<0.05\right.$ versus control). 
osteogenesis imperfecta or Ehlers-Danlos. ${ }^{17-19}$ Other previous work has also demonstrated reduced collagen I/III ratios in the skin and fascia taken from the scar tissue of patients with incisional hernias and patients with recurrent incisional hernias compared with that of healthy tissue or scar from patients without hernias., ${ }^{2,13}$ Therefore, the understanding of the pathogenesis of incisional and recurrent incisional hernia formation is based on the concept of abnormal collagen metabolism either through impaired wound healing or impaired constitutive collagen expression and formation associated with genetic disorders.

To our knowledge, this study is the first to examine nonscarred skin and fascial tissues of healthy control subjects and compare them with nonscarred skin and fascial tissues of patients with recurrent incisional hernias. This proved feasible by examining a patient population undergoing laparoscopic surgery, in which trocar incisions are typically made well away from the site of prior surgery or known pathology. This experimental design allowed us to compare primary, nonscarred tissue in both the experimental and control groups. The significant differences found in skin collagen I/III ratios between the two groups strongly suggest that the abnormal collagen metabolism of patients with recurrent incisional hernias is not only the result of abnormal wound healing as suggested in prior studies, but is also the result of an impaired constitutive collagen expression and formation.

In the present study, significant differences were found in the collagen I/III ratios between our experimental and control groups for skin, but not for fascial tissues. Because our power calculations were based on previous work, which compared nonscarred and scarred tissues and found larger ratio differences, ${ }^{2}$ it is possible the lack of a significant difference found in fascial tissues between groups may simply be the result of a type II error. However, nearly all previous work demonstrating alteration in collagen I/III ratios in primary tissues (e.g., osteogenesis imperfecta) have done so using skin tissues or fibroblasts cultured from dermal tissues. ${ }^{17-19}$ Therefore, the findings in this study may indicate that no significant difference exists in the primary fascial collagen ratio between groups.

Possible weaknesses in our study design may have potentially affected or confounded our results. Our study population was not stratified by hernia size, location or number of recurrences. Also, although the frequency of obesity in each group was the same, over 60 per cent of all patients were obese (mean body mass index $=34.5 \mathrm{~kg} / \mathrm{m}^{2}$ ). Obesity is thought to be a risk factor for hernia recurrence as a result of poorer wound healing, increased intra-abdominal pressure, and a technically more difficult surgical repair. ${ }^{20}$ Moreover, not all control subjects had clearly demon- strated they were not prone to hernia formation themselves, because 36 per cent had no abdominal surgical history before their enrollment in this study. However, on subanalysis of only those control subjects with prior abdominal surgical history and no hernia, our results were not significantly altered.

Our findings suggest a possible pre-existing collagen defect in the primary tissues of the abdominal wall in patients with recurrent incisional hernias. We believe that further research using microarray technology may elucidate a specific threshold or "protein signature" that could allow clinical screening for hernia formation risk. Such screening, if developed, could allow tailoring of future patients' surgical treatment so as to minimize or avoid the risk of incisional hernia.

\section{Acknowledgments}

We thank Kent van Sickle, M.D., for assistance in tissue collection and Dianne Lawson, Emory University Hospital Department of Pathology, for mounting and staining our biopsy specimens.

\section{REFERENCES}

1. DeFrances C, Hall M. 2005 National hospital discharge survey. Advance Data From Vital and Health Statistics 2007;385:16.

2. Klinge U, Si Z, Zheng H, et al. Collagen $\mathrm{I} / \mathrm{III}$ and matrix metalloproteinases (MMP) 1 and 13 in the fascia of patients with incisional hernias. J Invest Surg 2001;13:47-54.

3. Luijendijk R, Hop W, van-den-Tol M, et al. A comparison of suture with mesh repair for incisional hernia. N Engl J Med 2000; 343:392-8.

4. Zheng H, Si Z, Kasperk R, et al. Recurrent inguinal hernia: disease of the collagen matrix? World J Surg 2002;26:401-8.

5. Van-der-Rest M, Garrone R. Collagen family of proteins. FASEB J 1991;5:2814-23.

6. Fleischmajer R, Perlish JS, Burgeson RE, et al. Type I and type III interactions during fibrillogenesis. Ann N Y Acad Sci 1990;580:161-75.

7. Friedman DQ, Boyd C, Mackenzie JW, et al. Regulation of collagen gene expression in keloids and hypertrophied scars. J Surg Res 1993;55:214-22.

8. Henkel W, Glanville RW. Covalent crosslinking between molecules of type I and III collagen. Eur J Biochem 1982;122: 205-13.

9. Uitto J, Perejda AJ, Patrick-Abergel R, et al. Altered steadystate ratio of type I/III procollagen mRNAs correlates with selectively increased type I procollagen biosynthesis in cultured keloid fibroblasts. Proc Natl Acad Sci USA 1985;82:5935-9.

10. Romanic AM, Adachi E, Kadler KE, Hojima Y, Prockop DJ. Copolymerization of pNcollagen III and collagen I. pNcollagen III decreases the rate of incorporation of collagen I into fibrils, the amount of collagen I incorporated, and the diameter of the fibrils formed. J Biol Chem 1991;266:12703-9.

11. Birk DE, Mayne R. Localization of collagen type I, III, and $\mathrm{V}$ during tendon development. Changes in collagen types I and III are correlated with changes in fibril diameter. Eur J Cell Biol 1997;72:352-61. 
12. Dale PD, Sherratt JA, Maini PK. A mathematical model for collagen fibre formation during foetal and adult dermal wound healing. Proc R Soc Lond B Biol Sci 1996;263:653-60.

13. Klinge U, Si Z, Zheng $\mathrm{H}$, et al. Abnormal collagen I to III distribution in the skin of patients with incisional hernia. Eur Surg Res 2000;32:43-8.

14. Klinge U, Zheng H, Si Z, et al. Expression of the extracellular matrix proteins collagen I, collagen III and fibronectin and matrix metalloproteinase- 1 and -13 in the skin of patients with inguinal hernia. Eur Surg Res 1999;31:43-8.

15. Rosch R, Junge K, Knops M, et al. Analysis of collageninteracting proteins in patients with incisional hernias. Langenbecks Arch Surg 2003;387:427-32.

16. Rasband WS. Image J. 2004. Available at: http:\|rsb.info .nih.gov/ij. Accessed April 1, 2007.
17. Deak SB, Ricotta JJ, Mariani TJ, et al. Abnormalities in the biosynthesis of type III procollagen in cultured skin fibroblasts from two patients with multiple aneurysm. Matrix 1992;12: 92-100.

18. Rowe DW, Shapiro JR, Piorier M, Schlesinger S. Diminished type I collagen synthesis and reduced alpha 1(I) collagen messenger RNA in cultured fibroblasts from patients with dominantly inherited (type I) osteogenesis imperfect. J Clin Invest 1985;76:604-11.

19. Leim MSL, Van der Graff Y, Beemer FA, Van Vroonhoven TJMV. Increased risk for inguinal hernia in patients with EhlersDanlos syndrome. Surgery 1997;122:114-5.

20. Sauerland S, Korenkov M, Kleinen T, et al. Obesity is a risk factor for recurrence after incisional hernia repair. Hernia 2004;8: $42-6$. 
Copyright of American Surgeon is the property of Southeastern Surgical Congress and its content may not be copied or emailed to multiple sites or posted to a listserv without the copyright holder's express written permission. However, users may print, download, or email articles for individual use. 